Research Article

\title{
Detection and Elimination of Bathymetric Outliers in Multibeam Echosounder System Based on Robust Multi-quadric Method and Median Parameter Model
}

\author{
Shengping Wang ${ }^{1,2, *}$, Ping Zhou ${ }^{3}$, Ziyin $\mathrm{Wu}^{1}$, Jiabiao $\mathrm{Li}^{1}$ and Yongqi Wei ${ }^{4}$ \\ ${ }^{1}$ Key Laboratory of Submarine Geosciences, Second Institute of Oceanography, Hangzhou 310012, China \\ ${ }^{2}$ Faculty of Geomatics, East China University of Technology, Nanchang 330013, China \\ ${ }^{3}$ College of Marine Science and Technology, China University of Geosciences, Wuhan 430074, China \\ ${ }^{4}$ School of Civil and Environmental Engineering, University of California, Los Angeles, California 951593, USA
}

Received 24 March 2018; Accepted 28 June 2018

\begin{abstract}
Multibeam echosounder system is a dynamic measurement under the continuous motion of measurement platform. Contaminated sporadic outliers are inevitably generated during bathymetric data acquisition due to the interference of underwater environmental effects, such as ocean waves, wind, and tides. A filtering model of robust multi-quadric method based on median parameter was proposed in this study to detect and eliminate the outliers in bathymetric datasets. The submarine topography trend surface model was constructed by the multi-quadric function. Known node parameters in the function were obtained by the threshold extraction method of joining angle, chord with dispersion. The robust iterations of residual error initials and the weight-weakening process of outliers were studied by median parameters and IGGIII equivalent weight function. The validity of the proposed filtering model was verified by experimental datasets of offshore waters, which were collected by BV5000 multibeam side scan sonar. Results demonstrate that the known node selection strategy in this study can remove redundancy information in point set and extract regional characteristic points to the maximum extent. The fitting errors at normal bathymetric points are all below $0.06 \mathrm{~m}$, which are decreased compared with the corresponding magnitude of other schemes $(0.16 \mathrm{~m}, 0.12 \mathrm{~m})$. The residual initial value of robust iteration based on median parameters exhibites relatively high estimation accuracy and detection efficiency. The errors of bathymetric points are relatively small $(0.019 \mathrm{~m})$, and the detection number of outliers $(150)$ is efficient in the entire detection region. This work can improve the accuracy and efficiency of underwater terrain detection and lay a foundation for later scientific research and marine exploration.
\end{abstract}

Keywords: Bathymetry outlier detection, Multi-quadric function, Median parameter method, Robust estimation

\section{Introduction}

The availability of reliable bathymetry data has been of significant implications for marine applications, such as marine environment protection, offshore development and management, marine navigation, and submarine science [15]. Multibeam echosounder system (MBES) is the most widely employed in underwater topographic detection equipment at present. This system is a highly integrated set of various technologies, such as computer technology, navigation positioning technology, and digital sensor. According to the round-and-trip beam signals and data information of the auxiliary sensor, MBES can acquire a dataset with high accuracy, high density, and full-coverage sounding points [6-7]. Nevertheless, the detection process has abundant instable factors due to harsh underwater measurement conditions and the compatibility and comprehensiveness of combined subsystem, thereby resulting in differences between measured and actual depths in some places. Inaccurate detection and filtering may affect the accuracy of measurements.

*E-mail address: 37452116@163.com

ISSN: $1791-2377$ @ 2018 Eastern Macedonia and Thrace Institute of Technology. All rights reserved. doi:10.25103/jestr.113.10
The effects of bathymetric data are generally a comprehensive process. The real-time navigation positioning information, posture and sound velocity data, and echo signals of vessels should be edited or corrected to recognize and reject false signals and to recover and retain real information for improving the sounding accuracy and ensuring the drawing quality of seafloor topographic map [89]. These steps are essential preparations to the subsequent data processing and mapping control. In particular, the production of electronic navigational charts (ENCs) requires that the density of bathymetry data should be reduced to a certain extent to save storage space [10].

In addition to the influences of occasional errors, the collected swath sounding data often result in bathymetry outliers due to installation deviation, acoustic line tracking, vessel posture, and complex hydrological conditions in sea area [11-13]. These individual errors increase the accumulated total error budget of soundings. The quality of modified bathymetric data may not meet the international hydrographic organization normative requirements (IHO 2008) of seafloor mapping, although these errors have been corrected accordingly. Therefore, abnormal detection and filtering of bathymetric data must be executed accurately [14-15].

To this end, the purpose of this study was to provide an automatic filtering method for detecting outliers in 


\section{Journal of Engineering Science and Technology Review 11 (3) (2018) 70-78}

soundings. The trend surface of seabed was constructed by the multi-quadric (MQ) function, and a robust bathymetric estimation model was constructed through continuous iteration of equivalent weights. Representative characteristic points in the study area were selected according to multiple threshold criteria to provide parameters of known nodes for the combined equation in the MQ function. The median parameter model was applied to provide the optimal initial value of residual error in the iterative robust estimation. Therefore, the performance of overall observation equation contaminated by bathymetry outliers was reduced.

\section{State of the art}

The traditional interactive drawing generally processes abnormal bathymetric data by manual edition, which decreases operation efficiency and increases time consumption significantly. Early automation methods have realized the judgment and rejection of swath Ping space according to the threshold criteria, such as depth threshold, beam angle, and gradient [16]. Later, many experts have proposed a number of methods based on the statistical characteristics of neighborhood soundings, such as Cop, Ware, and Du's methods [17-19]. Although these methods are easy to operate, the parameters in these filter models need to be artificially set, and the degree of automation is not high. Huang and Vojinovic et al. applied the classification theory of support vector machine (SVM) into the detection of bathymetry outliers and recognized calibration of abnormal values accurately by constructing a continuously changing topological trend surface, which avoided annihilation of effective seabed topography [20-21]. Dong et al. proposed the filtering method of local trend surface based on polynomial fitting (PF) [22]. However, this method was only applicable to flat terrain and required blocking process of the survey area. This method led to incomplete filtering under complicated and continuously changing topography. In addition, this method lacked of robustness, and the presence of outliers might interfere with the overall operation performance and decrease the accuracy of abnormal detection.

Therefore, many studies on robust estimation and applications of robust filter have been reported to reduce the local outlier pollution of the entire bathymetric data. Debese et al. proposed a hierarchical adaptive robust method to build the seabed surface and separate out the sounding outliers [3]. This method was based on a parameter set analysis derived from space and quality, and the total surface outliers over $96.6 \%$ of the total surface could be processed in the study areas of Lexartec Rock Belt. Calder et al. combined the median filter method with Kalman filtering to smooth multibeam sounding data [23]. They also proposed the combined uncertainty and bathymetry estimator (CUBE) algorithm. On the basis of Bayes dynamic linear model, Kalman filtering, and the theory of multiple estimations, the CUBE algorithm calculated the robust estimation values of grid bathymetric nodes, which was used to realize real-time and high-precision outlier removal [24]. The latest phase of improvement model was conducive to perform bathymetric estimation effectively on large-scale mass datasets under limited computing resources. The model was adaptive to different depths and sensor-driven data densities [25].
Rezvani et al. provided an automatic filtering method with robust $\mathrm{M}$ estimation, which calculated the estimated value of each grid point by iterative derivation, and obtained the corresponding residual to discriminate outliers [26]. The model adopted segmented processing to mitigate the adverse effects of outlier observations. The reliability and working efficiency of this method verified the simulation and actual datasets. Chen et al. proposed the adaptive robust estimation based on least square (ARELS) [27]. However, this algorithm did not fully consider that using the least square estimation as the initial value might weaken the equivalent weight robust estimation.

On the basis of the above robust estimation methods, the topographic trend surface was constructed by polynomial surface fitting, which could not realistically close to the seabed topography. Besides, the key of robust estimation based on equivalent weight was to select the initial value with robustness and appropriate equivalent weight function [28-29]. Moreover, the median parameter model [30] exhibited strong robustness, and the MQ function [31] fitted to the characteristics of complicated topographic changes. As a result, a robust multi-quadric method based on median parameter (RMQMP) was proposed to detect bathymetry outliers generated from the process of multibeam sounding. First, the base of seabed terrain was constructed by the MQ function. Second, the threshold restriction method of joining angle, chord with dispersion (JACD) [32] was given to extract and obtain the known nodes of MQ function in the process of solving the initial value equations. Third, the initial value of robust iteration was calculated on the basis of median parameter model and the least squares criterion. Finally, the residual errors of different observations and corresponding equivalent weights were calculated according to robust iterative estimation based on IGGIII equivalent weight function [33], thus realizing detection and automatic filtering of abnormal values.

The remainder of this study is organized as follows. Section 3 introduces the research process of the proposed method and the detailed contents of modular algorithms. Section 4 presents the conducted experiment on measured bathymetric data. Contrast analysis and discussions of three schemes are performed. A series of indicators are used to evaluate and verify the credibility, validity as well as detection accuracy, and efficiency of the proposed algorithm. Section 5 summarizes the advantages of the designed method and what remains to be studied.

\section{Methodology}

Existence of bathymetric outliers may change the seabed topographic relief, which causes misjudgment on underwater topographic mapping and interferences in seabed exploration and scientific research significantly [34]. RMQMP method is accordingly proposed to solve the complex area of seabed topography. The module part of the algorithm mainly includes the MQ functional model of seabed topographic trend surface, known node selection strategy in MQ function, initial value acquisition of residual error based on median parameter model, and adaptive iterative process of equivalent robust estimation. The specific research route is shown in Fig. 1. 


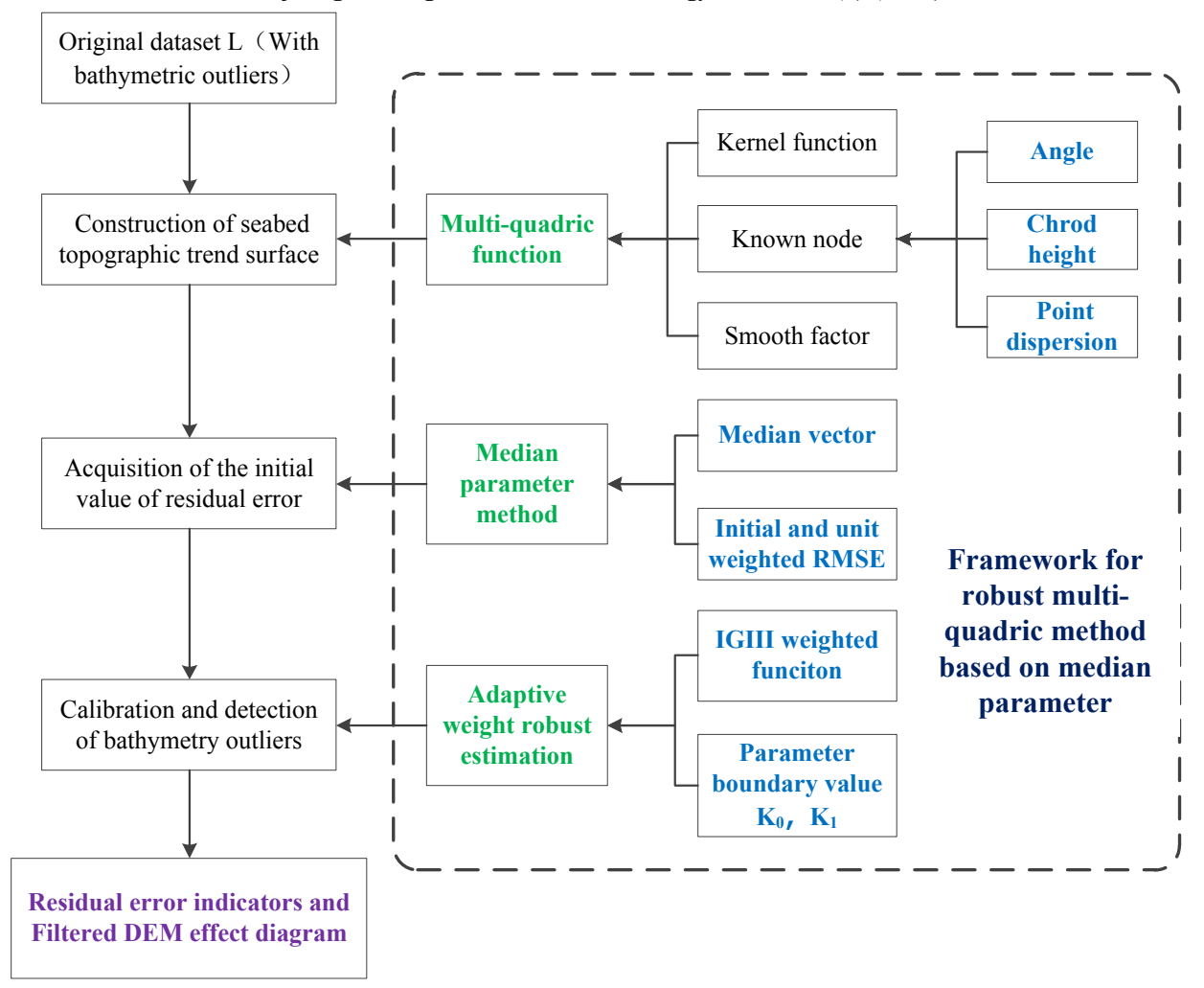

Fig. 1. Bathymetry Outlier Detection Procedure of RMQMP method

\subsection{Construction of basic function}

For complicated topography, PF model often can not reflect the real seabed topographic changes accurately. Through its constructed surface, using the fitting residual estimations as initial values for robust iteration has a high collapse rate. However, the MQ function establishes a functional relationship between each sampling point and all known nodes. Contributions of known nodes to each sampling point are iterated to represent the best surface fitting value and conform to the real topography [31]. One Ping bathymetric data are taken as an example, and PF and MQ method are used to fit them respectively, as shown in Fig. 2.

Therefore, the MQ model is used as a basis function to construct the regional seabed terrain, and the fitting equation is:

$$
Z(x, y)=\sum_{i=1}^{n} C_{i} A_{i}\left(x, y, x_{i}, y_{i}\right)
$$

\section{Where}

$Z(x, y)=$ water depth value of fitting area,

$(x, y)=$ coordinates of actual sampling points,

$\left(x_{i}, y_{i}\right)=$ coordinates of known points,

$n=$ number of known nodes,

$A_{i}=$ kernel function, and

$C_{i}=$ undetermined coefficient, i.e., it represents the contribution of $i-t h$ kernel function to the multilayer curve surface.

The selection of kernel function is usually symmetric, i.e.,

$$
A\left(x, y ; x_{i}, y_{i}\right)=\left[\left(x-x_{i}\right)^{2}+\left(y-y_{i}\right)^{2}+\delta\right]^{\beta}
$$

Where

$\delta=$ smooth factor, and

$\beta=$ power index, usually election as $-0.5,0.5$, and 1.5 . In this paper, smooth factor $\delta=1 \mathrm{e}-6$, power index of kernel function $k=0.5$.

The central beam area data collected by transducer is of high accuracy and reliability in general. Therefore, several adjacent swath depth data from central beam area are selected, which represent a total of $m$ observations, of which $\mathrm{n}$ denotes known nodes (for its selection, see Subsection 3.2). The matrix expression of Formula (1) is:

$\left[\begin{array}{c}Z_{1} \\ Z_{2} \\ \mathrm{M} \\ Z_{m}\end{array}\right]=\left[\begin{array}{cccc}A_{11} & A_{12} & \mathrm{~L} & A_{1 n} \\ A_{21} & A_{22} & \mathrm{~L} & A_{2 n} \\ \mathrm{M} & \mathrm{M} & \mathrm{M} & \mathrm{M} \\ A_{m 1} & A_{m 2} & \mathrm{~L} & A_{m n}\end{array}\right] \mathrm{g}\left[\begin{array}{c}C_{1} \\ C_{2} \\ \mathrm{M} \\ C_{n}\end{array}\right] \Rightarrow Z=A C$

According to the principle of least squares criterion, when $m$ (number of observations) is not less than $n$ (number of known nodes), the equation of fitting residual error is:

$V=A C-Z \Rightarrow C=\left(A^{T} P A\right)^{-1} A^{T} P Z$

The accuracy evaluation of residual error is:

$\sigma=\sqrt{[v v] /(m-1)}$

Where

$\sigma=$ root mean square error (RMSE), and

$v=$ difference between the fitting and measuring bathymetric data. 

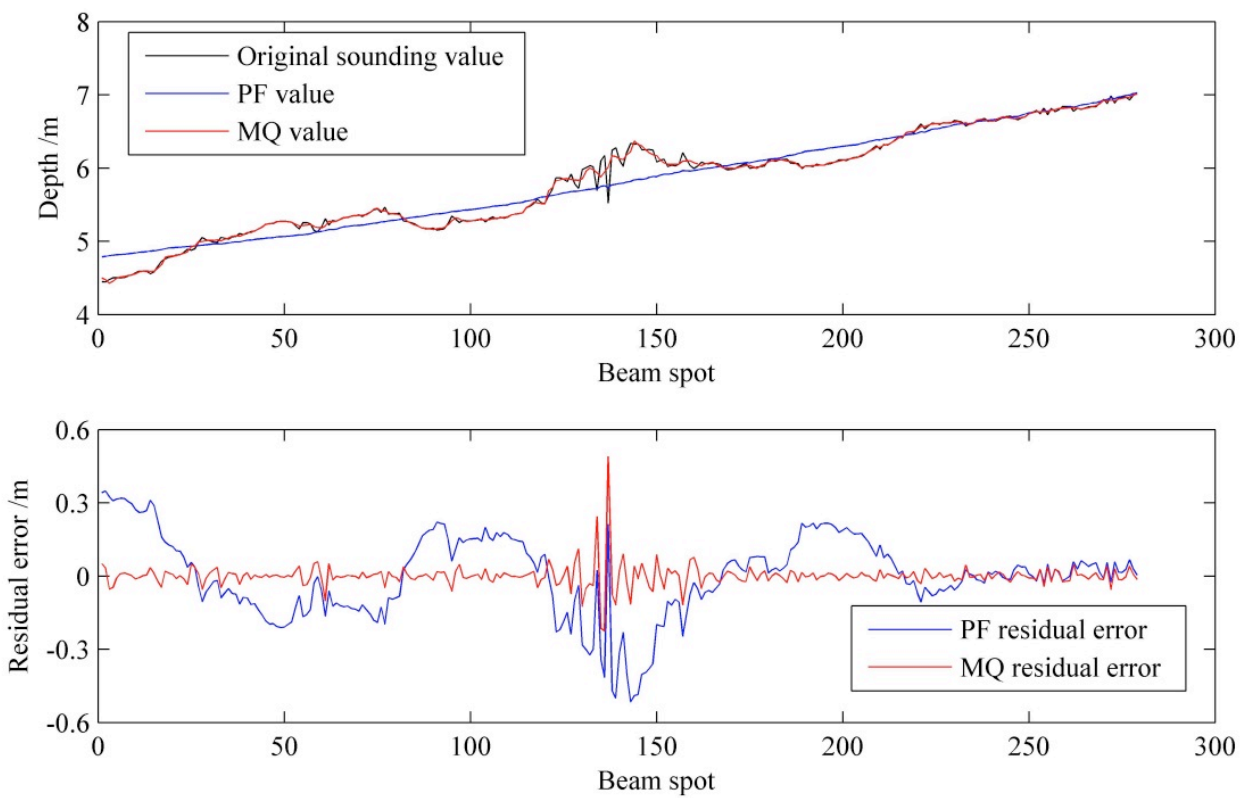

Fig. 2. Sounding fitting errors of 1 Ping bathymetry data under PF and MQ model

\subsection{Selection of known nodes}

Multibeam swath bathymetric data have the characteristics of mass size and dense point distribution. Three key problems, namely, kernel function, smooth factor, and node position, have to be solved when selecting MQ model as the primary function to construct the surface. Among them, node position is the most difficult to be determined. Nodes are traditionally calculated by equal distance or equal interval [35]. The gained nodes have high data redundancy and repetitive rate. Some nodes are vulnerable to be contaminated by abnormal bathymetric data. Therefore, the strategy of joining angle, chord with dispersion (JACD) is adopted to comprehensively extract the regional feature points and use them as known nodes in this study.

\subsubsection{Joint angle and chord criterion}

Observation points (or their spatial interpolation points), which are used as the known nodes, must be satisfied such that observation points are not points of outliers generated in the detection process. The joint angle and chord criterion is used to preliminary screen outliers for each Ping observation data and obtain relatively credible bathymetry points. The steps are as follows:

Step 1) the limited error of angle $(\Delta \alpha)$ and chord error threshold $(\Delta h)$, as shown in Fig. 3. The limited angle is $10^{\circ}$, and the chord threshold is $10 \%$ of the mean value of each Ping bathymetric data.

Step 2) From the starting point, the adjacent sounding points $P_{1}, P_{2}$ and $P_{3}$ are taken, the angle $(\alpha)$ between $\overline{P_{1} P_{2}}$ vector and $\overline{P_{2} P_{3}}$ vector is calculated, and the chord height $(h)$ between sounding point $P_{2}$ and $\overline{P_{1} P_{3}}$ vector is obtained.

Step 3) whether $\alpha>\Delta \alpha$ and $h>\Delta h$ are true is judged. If yes, $P_{2}$ is marked abnormal point. Meanwhile, $P_{1}=P_{1}$, $P_{2}=P_{3}$, and $P_{3}=P_{4}$ are set. If not, $P_{2}=P_{1}, P_{2}=P_{3}$, and $P_{3}=P_{4}$ are set. Whether $\alpha$ and $h$ are higher than the corresponding threshold is continuously judged, and the abnormal value is calibrated until observation points in this Ping data are processed. Similar processing is performed in the next Ping data. Finally, reliable observation points in the region after preliminary screening are gained.

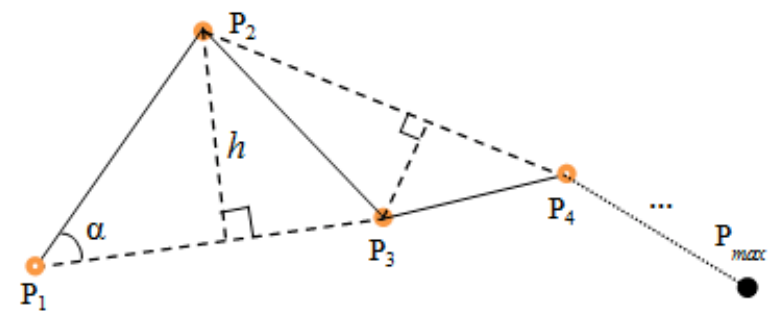

Fig. 3. Schematic of joint angle and chord criterion

\subsubsection{Dispersion of point}

The above preliminarily selected reliable points will form a certain area, among them, the bathymetric observation value of one point is set $z\left(x_{i}, y_{i}\right)$, and $\bar{z}$ is the mean bathymetric value of all points in the region. Therefore, dispersion of this point in the region can be defined as [32]:

$$
D\left(x_{i}, y_{i}\right)=\frac{\left(z\left(x_{i}, y_{i}\right)-\bar{z}\right)}{\left\{\left(\sum_{i=1}^{k}\left(z\left(x_{i}, y_{i}\right)-\bar{z}\right)+\varepsilon\right) / k\right\}}
$$

where

$\varepsilon=$ a small positive value and is used to prevent situation of zero denominator, and

$k=$ the total number of regional trusted sampling points.

Based on the concept of point dispersion, the above credible observation points are treated by sparse processing, and the sparse feature points are used as known nodes of MQ function. The thinning process is as follows:

Step 1) The threshold of height difference and the minimum distance limit among points are given. The threshold of dispersion is $150 \%$ of water depth.

Step 2) Reliable observation points are arranged in a proper order according to bathymetric values. If the difference in extreme bathymetric values of observation 
Shengping Wang, Ping Zhou, Ziyin Wu, Jiabiao Li and Yongqi Wei/

Journal of Engineering Science and Technology Review 11 (3) (2018) 70-78

points is higher than the dispersion threshold, the maximum and minimum points are used as candidate points of topographic feature and are reserved. Dispersion of other points in the computing region is calculated. Points with dispersion higher than the threshold are reserved markup, otherwise, they will not be processed.

Step 3) The adjacent region is searched according to marked dispersion points. The points in the adjacent domain close to the current candidate characteristic point and with dispersion smaller than the threshold are deleted, and the rests comprise the characteristic point set.
Dispersion of point reflects the contribution of this point to regional topographic relief [32]. Therefore, points with great contributions to topographic relief (positive and negative) in the evacuation process are retained, which is conducive to maintain topographic relief features in the survey area. Fig. 4 shows that the equal interval of 4 to 1 is compared with the proposed algorithm, and residual errors after MQ fitting are used to evaluate the accuracy. The diagram shows that corrected residual errors of the proposed algorithm are closer to the middle value 0 for the normal sounding values, and the outstanding depth position value can be more prominent for the bathymetry outliers.

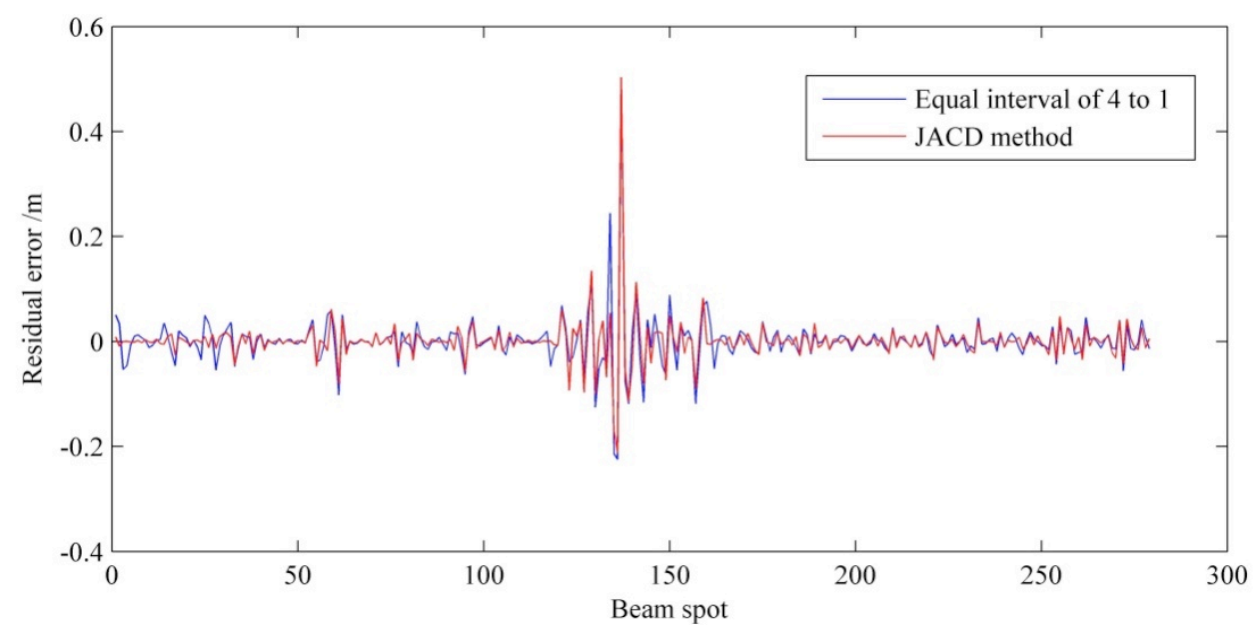

Fig. 4. Residual error values of different selection methods about known nodes

Note: Residual errors of 1 Ping data after fitting for protruding the change in regional detail features.

\subsection{Calculation of robust iterative initial values}

Median parameter method is used when constructing a terrain trend surface by the MQ model as a basis function to solve the model parameter coefficients in Equation (4), i.e., the contribution value of each known node $\mathrm{Ci}$. Subsection 3.2 shows that $\mathrm{n}$ known nodes in MQ function are obtained. In the observation Equation (4), $(n+1)(m>(n+1))$ equations are selected randomly from $m$ observation equations. Under the premise that the accuracy of parameter estimation can be guaranteed, the parameter estimation vector $C$ is solved, which yields $p=C_{m}^{n+1}$ groups of combined solutions. The $i-t h$ model parameter value $c_{i}$ is reconstructed into $p \times 1$ dimensional vector as:

$\hat{c^{i}}=\left[\begin{array}{c}\hat{c_{1}^{i}}, \hat{c_{2}^{i}}, \mathrm{~L}, \hat{c}_{p}^{i} \\ \end{array}\right]$

The median of the parameters (median $\left(c^{i}\right)$ ) is achieved in Formula (7). The other model coefficients are similar. Finally, the median vector of the constituent model parameters is $\hat{C}_{\text {med }}$,

$\hat{C}_{\text {med }}=\left[\operatorname{median}\left(\hat{c}^{1}\right)\right.$, median $\left(\hat{c}^{2}\right), \mathrm{L}$, median $\left.\left(\hat{c}^{n}\right)\right]$

The difference between different groups of solutions and median vector is calculated. For the $p$ groups of solutions, $\left\{\begin{array}{l}d c_{j}=C_{j}-\hat{C}_{\text {med }} \\ \min \_d c=\min \left\{\left\|d c_{1}\right\|,\left\|d c_{2}\right\|, \mathrm{K},\left\|d c_{p}\right\|\right\}\end{array}\right.$

where

$j=1 \mathrm{~L} p,\left\|d c_{j}\right\|=$ secondary norm of $d c_{j}$, and $\min \_d c=$ the minimum value of corresponding model parameter vector, i.e., the solution of median parameter method in this study.

Correction of residual error that corresponds to the $m \times 1$ dimension of the entire observation vector can be calculated from Equation (4). In this case, the residual error is used as the initial value of robust estimation. The mean square error of the initial unit weight $(\sigma 0)$ is recorded as:

$\left\{\begin{array}{l}v_{\text {med }}=\text { median }\left\{\left|v_{1}\right|,\left|v_{2}\right|, \mathrm{L},\left|v_{m}\right|\right\} \\ \sigma_{0}=v_{\text {med }} / 0.6745\end{array}\right.$

\subsection{Iterative estimation of equivalence weight}

The residual error matrix $V_{0}$ and the initial value of unit weighted error $\sigma_{0}$ in bathymetry data are obtained from the median parameter method in Subsection 3.3. On the basis of the principle of robust estimation $A^{T} \bar{P} V=0$, the iteration process of equivalent weight robust estimation is:

$\left\{\begin{array}{l}C^{(k+1)}=\left(A^{T} \bar{P}^{(k)} A\right)^{-1} A^{T} \bar{P}^{(k)} Z \\ V^{(k+1)}=A C^{(k+1)}-Z\end{array}\right.$ 
The equivalent weight matrix is:

$$
\bar{P}^{(k)}=\left[\begin{array}{cccc}
\bar{p}_{1}^{(k)} & & & \\
& \bar{p}_{2}^{(k)} & & \\
& & 0 & \\
& & & {\overline{p_{m}}}^{(k)}
\end{array}\right]
$$

Many types of equivalent weight functions exist. The IGIII weight function [36] is used to determine the weights for each sounding value in this study, i.e.,

$$
\bar{p}_{i}^{(k)}=\left\{\begin{array}{cc}
1 & \left|v_{i}^{\prime^{\prime}(k)}\right| \leq k_{0} \\
\frac{k_{0}}{\left|v_{i}^{\prime(k)}\right|\left[\frac{\left(k_{1}-\left|v_{i}^{\prime(k)}\right|\right)}{\left(k_{1}-k_{0}\right)}\right]^{2}} & k_{0} \leq\left|v_{i}^{\prime(k)}\right| \leq k_{1} \\
0 & \left|v_{i}^{\prime(k)}\right| \geq k_{1}
\end{array}\right.
$$

where

$$
\begin{aligned}
& \left|v_{i}^{(k)}\right|=v_{i}^{(k)} / \hat{\sigma}_{i}^{(k)} \text {, i.e., the standardized residual error, } \\
& \hat{\sigma}_{i}^{(k)}=\operatorname{median}\left(\left|v_{i}\right|^{(k)}\right) / 0.6745, \text { and } k_{0}=1.0 \text { to } 2.5 \text { and } k_{1}=
\end{aligned}
$$

3.0 to 8.0, which are from Literature [37]. However, the two parameters depend on the fluctuation of seabed topography actually.

The estimated parameters and corrections are calculated according to continuous iteration of Equation (11). The iteration termination condition is $\max _{i}\left|C_{i}^{k+1}-C_{i}^{k}\right| \leq \varepsilon$ ( $\varepsilon$ is a small positive). In the iteration process, weights of abnormal bathymetric points are decreased continuously, and the calculation accuracy of model coefficients is increased by changing the standardized residual error continuously [26]. Consequently, the abnormal bathymetric points can be recognized accurately. The bathymetric data when the weight is close to 0 after iteration termination are determined as the abnormal values.

\section{Result analysis and discussion}

The experimental data were obtained from the bathymetric data of a shallow sea area by the BV5000 multibeam side scan sonar. Ten Ping continuous data that can reflect detailed features significantly were selected as test data. Footprint points with poor beam accuracy at edges of different Ping were abandoned, and a total of 1904 bathymetric points were selected. The water depth ranged from $4 m$ to $8 m$ in the test area.

An MQ model was used as a basis function to construct the regional terrain trend surface, and the number of known nodes was obtained $(n=635)$ through the JACD algorithm. Considering this fact that the bathymetry outliers do not exceed $10 \%$ of the total number, moreover, the singleparameter median method's crash pollution rate is close to $50 \%$, that is, if more than half of the observations are normal, the median must be normal values [37]. Therefore, when the median parameter method was used to solve the initial value of robust estimation for fully improving the program efficiency, it determined $p=1000$ times to solve each parameter estimation vector $C_{i}$ and obtained the final median vector $\hat{C}_{\text {med }}$ and residual error initial matrix $V_{0}$. In addition, two parameter boundaries were selected as $k_{0}=3$ and $k_{1}=6$ in the robust iterative process. Unlike the performance and validity of other strategic schemes, those of the proposed algorithm were analyzed and verified from the perspectives of residual error and detection efficiency, as well as digital elevation model (DEM) effect graph.

\subsection{Residual error and detection efficiency}

Three schemes were used to analyze the detection precision of bathymetry outliers. The residual error, RMSE, and number of detection points after correction were used to verify the feasibility and advantages of this design method (Table. 1 and Fig. 5).

Scheme 1: traditional multi-quadric fitting (MQ),

Scheme 2: adaptive robust estimation based on least square (ARELS), and

\begin{tabular}{|c|c|c|c|c|c|c|c|}
\hline \multirow{2}{*}{ Method } & \multicolumn{3}{|c|}{ Residual of all points/m } & \multicolumn{3}{|c|}{ Residual of normal points / $m$} & \multirow{2}{*}{ Number of outlier } \\
\hline & Max & Min & RMSE & Max & Min & RMSE & \\
\hline MQ & 0.475 & -0.350 & 0.051 & 0.150 & -0.151 & 0.032 & 55 \\
\hline ARELS & 0.629 & -0.527 & 0.029 & 0.102 & -0.101 & 0.022 & 97 \\
\hline RMQMP & 0.504 & -0.390 & 0.019 & $\mathbf{0 . 0 5 3}$ & -0.054 & 0.013 & 150 \\
\hline
\end{tabular}

Scheme 3: robust multi-quadric method based on median parameter (RMQMP).

Table. 1. Evaluation of statistical parameters

Table. 1 and Fig. 5 indicated five main findings. First, compared with Scheme 1, Schemes 2 and 3 are robust estimation methods. For normal bathymetry points (Columns 5 to 7), their maximum and minimum values of residual error and RMSEs were very small, which implied that their fitting precision was close to the theoretical bathymetry values and conformed to their definition. Second, for the sounding points of the entire survey area (including outlier depth values, Columns 2 to 4 ), the maximum and minimum residual errors obtained after the iteration were prominent, and they are shown in Lines 3 to 4 of Tab. 1 . The plot map analysis presented that this part of outstanding points was mainly the bathymetry outliers and thus could better illustrate the superiority of robust estimation methods in Schemes 2 and 3. Third, unlike the RMSE obtained from Scheme 1, the smaller RMSEs obtained from Schemes 2 and 3 were below $0.03 \mathrm{~m}$, which provided a basis for determining the abnormal value. The detection efficiency was also improved. Fourth, from Columns 2 to 4 , unlike the residual values of outliers in Scheme 2 (least-square initial value), those in Scheme 3 (initial value of median parameter method) were not so obvious, but the abnormality was judged on the basis of whether the equivalence weights were close to 0 after iteration in the robust estimation. Fifth, from Column 8 and Line 4, the RMQMP method (Scheme 3) exhibited relatively high estimation accuracy and detection efficiency in terms of fitting residuals and the judgment of normal sounding points. 
(a). Fitting residuals based on MQ method
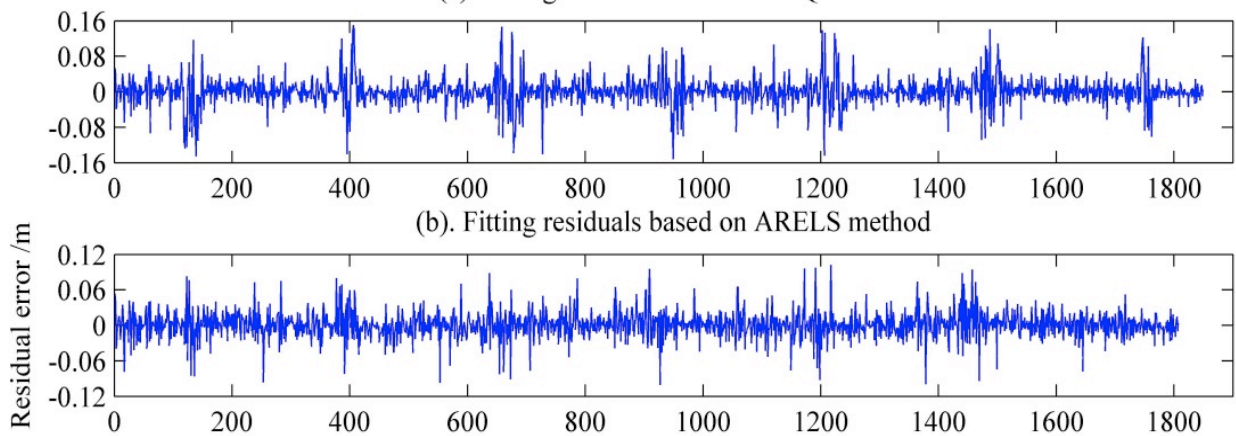

(c). Fitting residuals based on RMQMP method

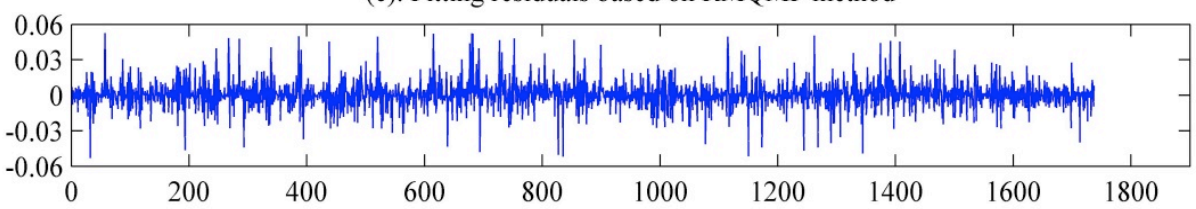

Fig. 5. Residual error values of normal sounding points

\subsection{DEM effect diagram}

First, the unprocessed multibeam bathymetric data were gridded, as shown in Fig. 6 (a). Afterward, the abnormal bathymetry data were eliminated by RMQMP method, and the remaining bathymetric data were gridded to generate the DEM, as shown in Fig. 6 (c). Finally, the robust multiquadric method based on the least squares initial value was compared, and its DEM effect is shown in Fig. 6 (b). Some prominent abnormal values exist at the center of Fig. 6 (b) This figure also has features of over filtering, such as the red frame, which determined the characteristics of the submarine undulations as the sounding outliers and filtered them out. Fig. 6 (c) displays that basically no abnormally prominent undulations occurred, and the features of the submarine details with topographic changes were preserved in measurement area.
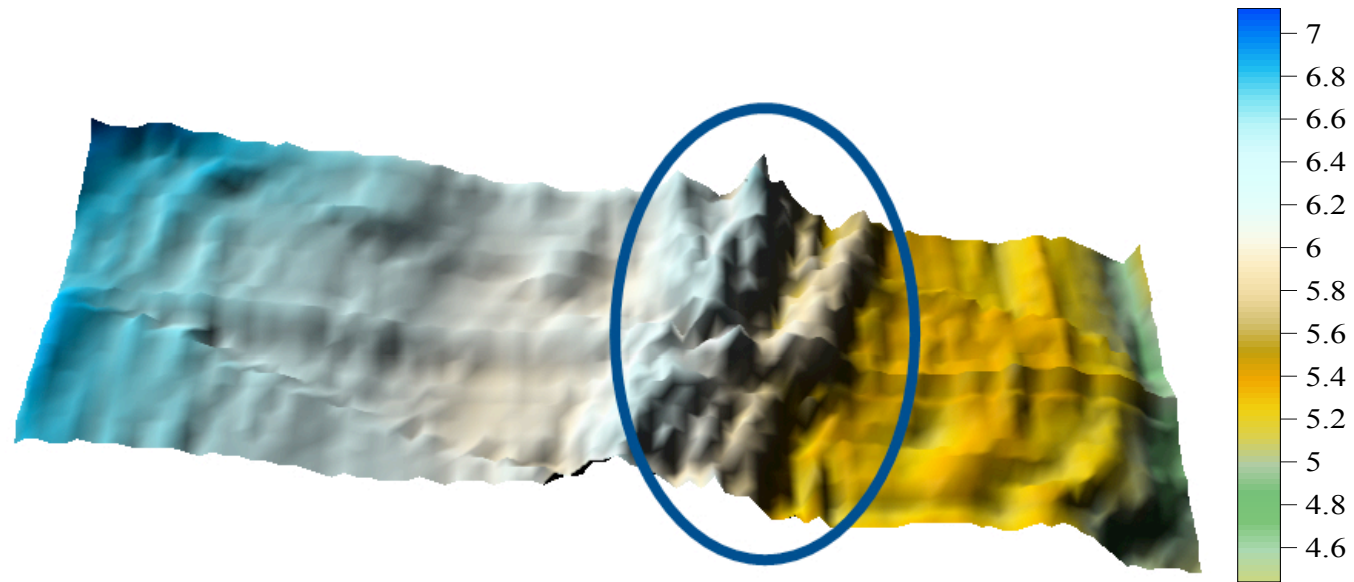

(a) Unfiltered original area DEM

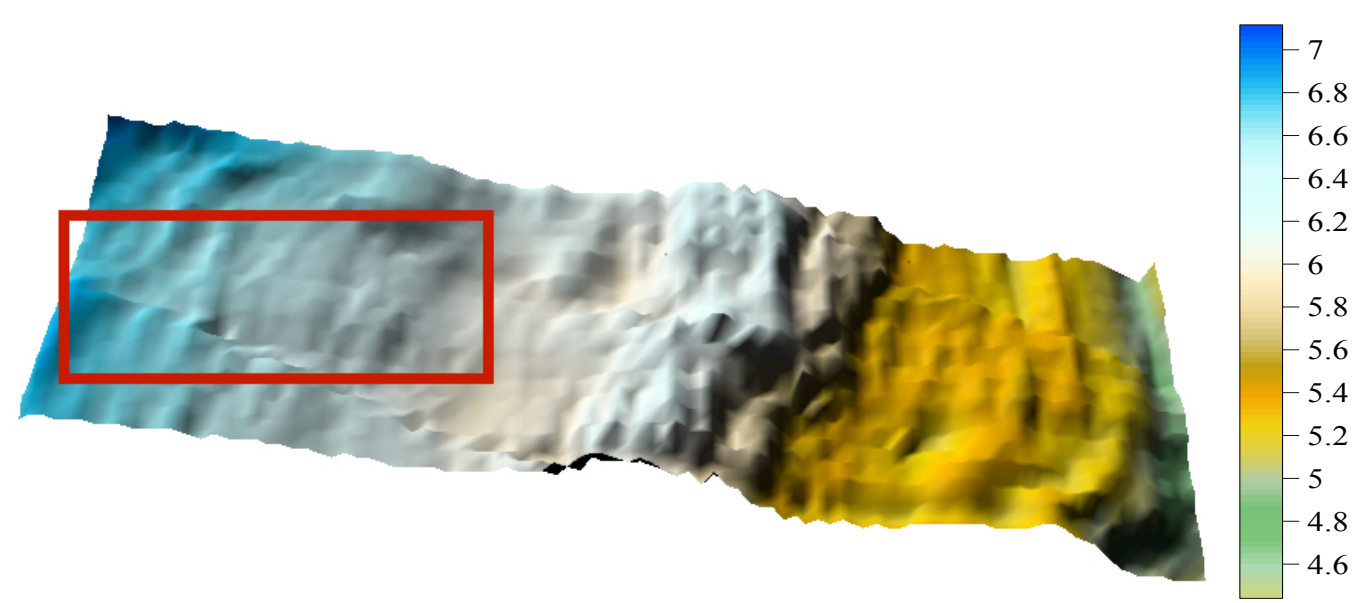

(b) DEM corrected by robust multi-quadric method based on the initial value of least squares 


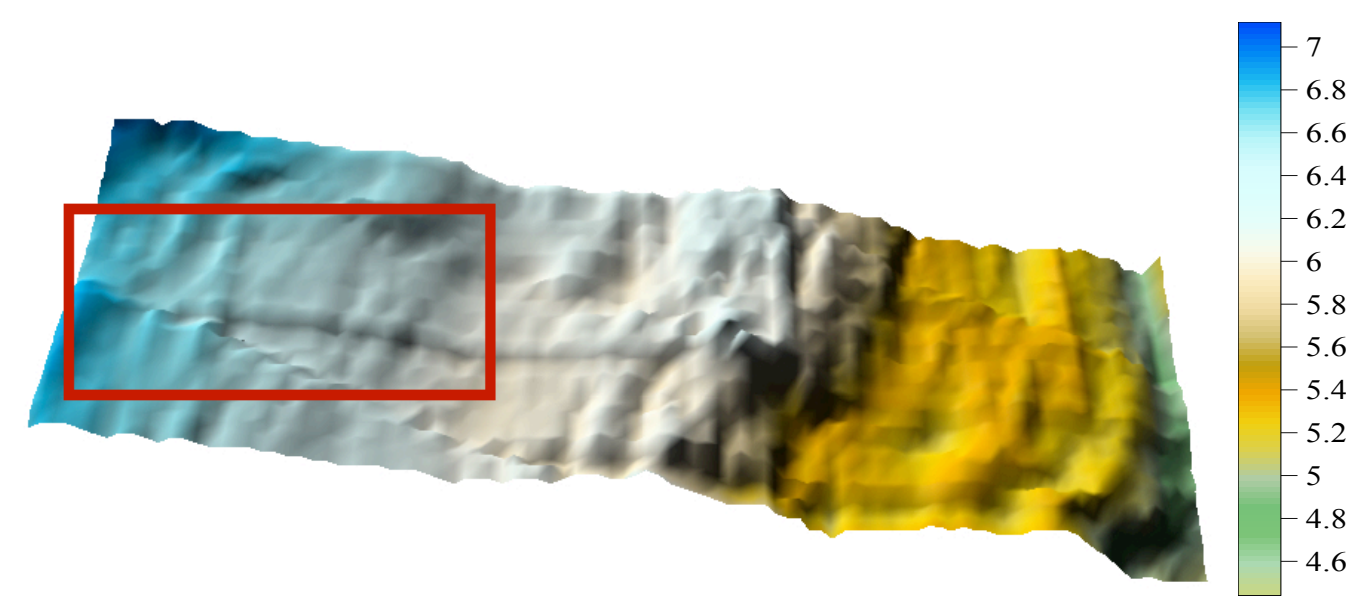

(c) DEM corrected by RMQMP model

Fig. 6. Original and corrected surveyed area DEM

\section{Conclusions}

In order to improve the detection efficiency and accuracy of bathymetry outliers in multibeam sounding data, this study started with the construction of the fitting trend surface model. And the median parameter method was used to obtain the initial value of residual error in the robust iteration for weakening the weight of outliers and the overall contamination. As a result, a robust estimation of regional water depth was achieved, and bathymetry outliers were eliminated. Bathymetric data were estimated by combining the difference between residual error and mean square error, the number of abnormal detection, and the DEM effect diagram after correction by different schemes. Some major conclusions could be drawn.

(1) The JACD method can remove the redundancy information points and coarse error bathymetric points to the maximum extent. The characteristic changing points of multibeam bathymetric data are retained through the secondary dilution. Therefore, the simplified feature points can basically meet the requirements of MQ function fitting and improve the operation efficiency, which lays the foundation for subsequent robust estimation to eliminate abnormal values.

(2) When the residuals are calculated on the basis of least squares as the initial values, the residual errors are easy to be contaminated by the outliers in the solution process and often can converge to the ideal extremum, which affects the performance of subsequent robust estimation. However, the median parameter method assures the robustness of parameter estimation when solving MQ model parameters. The acquired residual values are optimal at this time. The experiment proves that using this residual error as the robust initial error can inhibit the abnormal values in equivalent weight robust iteration.
This study concluded from the combination of theoretical research and actual dataset that the initial value of robust iterative estimation played an important role. The constructed initial value solving model could weaken the contamination effect of outliers and achieved satisfying detection efficiency and filtering accuracy of abnormal multibeam bathymetric data. The integrated RMQMP method achieved good results in the detection efficiency and accuracy of bathymetry outliers, which could provide accurate judgment references and reliable underwater information in follow-up marine scientific studies, such as coastal engineering and continental shelf structure. However, many aspects, such as the limited bathymetric range, different types of weight functions, and the setting parameters of each sub module, need to be further studied. Future studies will make further explorations and contrast analysis to provide an integrated model that can recognize and eliminate abnormal bathymetric values more accurately and effectively.

\section{Acknowledgements}

This work was supported by the Open Fund Project of Jiangxi Provincial Digital Land and Environment Laboratory (DLLJ201719), the Fund Project of Jiangxi Provincial Education Department (GJJ170447), the Natural Science Foundation of Jiangxi Province in China (20161BAB 206163), the National Natural Science Foundation of China (41206078), and the Director Fund of Second Institute of Oceanography (JG-1508).

This is an Open Access article distributed under the terms of the Creative Commons Attribution License

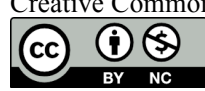

\section{References}

1. Davies, J. S., Howell, K. L., Stewart, H. A., et al., "Defining biological assemblages (biotopes) of conservation interest in the submarine canyons of the South West Approaches (offshore United Kingdom) for use in marine habitat mapping". Deep-Sea Research Part II, 104(2), 2014, pp.208-229.
2. Pendleton, E. A., Brothers, L. L., Thieler, E., et al., "Sand ridge morphology and bedform migration patterns derived from bathymetry and backscatter on the inner-continental shelf offshore of Assateague Island, USA", Continental Shelf Research, 144, 2017, pp.80-97.

3. Debese, N., MoitiÉ, R., Seube, N., "Multibeam echosounder data cleaning through a hierarchic adaptive and robust local surfacing". Computers \& Geosciences, 46(3), 2012, pp.330-339. 
4. Ernstsen, V. B., Noormets, R., Hebbeln, D., et al., "Precision of high-resolution multibeam echo sounding coupled with highaccuracy positioning in a shallow water coastal environment". GeoMarine Letters, 26(3), 2006, pp.141-149.

5. Bastia, R., Radhakrishna, M., Nayak, S., "Identification and characterization of marine geohazards in the deep water eastern offshore of India: constraints from multibeam bathymetry, side scan sonar and 3D high-resolution seismic data". Natural Hazards, 57(1), 2011, pp.107-120.

6. Cutter, G. R., Berger, L., Demer, D. A., "A comparison of bathymetry mapped with the Simrad ME70 multibeam echosounder operated in bathymetric and fisheries modes". Ices Journal of Marine Science, 67(6), 2010, pp.1301-1309.

7. Fezzani, R., Zerr, B., Mansour, A., et al., "Fusion of Swath Bathymetric Data: Application to AUV Rapid Environment Assessment”. IEEE Journal of Oceanic Engineering, 99, 2018, pp.1-10.

8. Ardalan, A. A, Rezvani, M. H., "Improved vessel squat modeling for hydrographic and navigation applications using kinematic GNSS positioning". Gps Solutions, 18(2), 2014, pp.253-262.

9. Yang, F. L., Li, J. B., Han, L. T., et al. "The filtering and compressing of outer beams to multibeam bathymetric data". Marine Geophysical Research, 34(1), 2013, pp.17-24.

10. Moszynski, M., Chybicki, A., Kulawiak, M., et al., "A novel method for archiving multibeam sonar data with emphasis on efficient record size reduction and storage". Polish Maritime Research, 20(1), 2013, pp.77-86.

11. Zirek, E., Sunar, F., "Change Detection Of Seafloor Topography By Modeling Multitemporal Multibeam Echosounder Measurements". In: The International Archives of the Photogrammetry, Remote Sensing and Spatial Information Sciences, Istanbul, Turkey: Gottingen: Copernicus GmbH, 2014, pp.221-227.

12. Talib, K. H., Othman, M. Y., Sulaiman, S. A. H., et al., "Determination of speed of sound using empirical equations and SVP”. In: 7th International Colloquium on Signal Processing and its Applications, Penang, Malaysia: IEEE, 2016, pp.252-256.

13. Zhao, J. H., Yan, J., Zhang, H. M., et al., "A new method for weakening the combined effect of residual errors on multibeam bathymetric data". Marine Geophysical Research, 35(4), 2014, pp.379-394.

14. Brekke, E., Hallingstad, O., Glattetre, J., "Tracking Small Targets in Heavy-Tailed Clutter Using Amplitude Information". IEEE Journal of Oceanic Engineering, 35(2), 2010, pp.314-329.

15. GarcãA, R. P., GarcãA, F. P., Ruiz, J. M. D. L., et al., "Model for the Processing and Estimation of Dual Frequency Echo Sounder Observations in Detailed Bathymetries". Marine Geodesy, 39(3-4), 2016, pp.305-320.

16. Bjørke, J. T., Nilsen, S., "Fast trend extraction and identification of spikes in bathymetric data". Computers \& Geosciences, 35(6), 2009, pp.1061-1071.

17. Perry, R., "Scientific and Hydrographic use of the Bathymetric SWATH Survey System". In: Oceans 82, Washington, DC, USA: IEEE, 1982, pp.396-401.

18. Ware, C., Knight, W., Wells, D., "Memory intensive statistical algorithms for multibeam bathymetric data". Computers \& Geosciences, 17(7), 1991, pp.985-993.

19. Du, Z., Wells, D., Mayer, L., "An approach to automatic detection of outliers in multibeam echo sounding data". Hydrographic Journal, (79), 1996, pp.19-23.
20. Huang, X. Y., Zhai, G. J., Sui, L. F., et al., "Application of Least Square Support Vector Machine to Detecting Outliers of Multibeam Data". Geomatics and Information Science of Wuhan University, 35(10), 2010, pp.1188-1191+1196.

21. Vojinovic, Z., Abebe, Y. A., Ranasinghe, R., et al. "A machine learning approach for estimation of shallow water depths from optical satellite images and sonar measurements". Journal of Hydroinformatics, 15(4), 2013, pp.1408-1424.

22. Dong, J., Ren, L., S., "Filter of MBS Sounding Data Based on Trend Surface". Hydrographic Surveying and Charting, China, 27(6), 2007, pp. 25-28.

23. Calder, B. R., Mayer, L. A., "Robust automatic multi-beam bathymetric processing". In: Hydrographic Conference. Norfolk, VA, USA: Hydrographic Society of America, 2001, pp.1-20.

24. Calder, B. R., Mayer, L. A., "Automatic processing of high-rate, high-density multibeam echosounder data". Geochemistry Geophysics Geosystems, 4(6), 2003, pp.24-28.

25. Calder, B. R., Rice, G., "Computationally efficient variable resolution depth estimation”. Computers \& Geosciences, 106, 2017, pp.49-59.

26. Rezvani, M. H., Sabbagh, A., Ardalan, A. A., "Robust Automatic Reduction of Multibeam Bathymetric Data Based on Mestimators". Marine Geodesy, 38(4), 2015, pp.327-344.

27. Chen, Z. H., Lu X. F., "Gross Error Elimination in DEM Data Based on Adaptive Robust Least Squares Estimation". Hydrographic Surveying and Charting, China, 26(6), 2006, pp.1517.

28. Yang, L., Shen, Y. Z., Lou, L. Z. "Equivalent Weight Robust Estimation Method Based on Median Parameter Estimates". Acta Geodaetica et Cartographica Sinica, 40(1), 2011, pp.28-32.

29. Tao, Y. Q., Gao, J. X., Yao, Y. F., "Solution for Robust Total Least Squares Estimation Based on Median Method". Acta Geodaetica et Cartographica Sinica, 45(3), 2016, pp.297-301.

30. Marks, K. M., Smith, W. H. F., "Detecting small seamounts in AltiKa repeat cycle data". Marine Geophysical Research, 37(4), 2016, pp.1-11.

31. Chen, F. C., Liu, F. Y., Yan, C. Q., et al., "A Huber-derived Robust Multi-quadric Interpolation Method for DEM Construction". Geomatics and Information Science of Wuhan University, 41(6), 2016, pp.803-809.

32. Zi G. F., Chen X. L., Liu Y., et al., "New Thinning Method of Multi-beam Bathymetric Data Based on Terrain Matching". The Ocean Engineering, China, 31(6), 2013, pp.118-123.

33. Koch, K. R., Yang, Y., "Robust Kalman filter for rank deficient observation models". Journal of Geodesy, 72(7-8), 1998, pp.436441.

34. Micallef, A., Bas, T. P. L., Huvenne, V. A. I., et al., "A multimethod approach for benthic habitat mapping of shallow coastal areas with high-resolution multibeam data". Continental Shelf Research, 39-40(2), 2012, pp. 14-26.

35. Wang, F. W., Zhou, S. J., Zhou, Q., et al. "Comparisons between three methods in initial residuals problem of selecting weight iteration method". Science of Surveying \& Mapping, China, 40(8), 2015, pp.18-21.

36. Li, H. J., Tang S. H., Huang J., "Discussion for the Selection of Constant in Selecting Weight Iteration Method in Robust Estimation". Science of Surveying and Mapping, China, 31(6), 2006, pp.70-72.

37. $\mathrm{Xu}, \mathrm{P}$. , "Sign-constrained robust least squares, subjective breakdown point and the effect of weights of observations on robustness". Journal of Geodesy, 79(4-5), 2005, pp.288-288. 